\title{
Trochlear Nerve Schwannoma: Case Report and Literature Review
}

\section{Schwannoma do nervo troclear: revisão da literatura e relato de caso}

\author{
Marcelo Lemos Viera da Cunha ${ }^{1,2}$ Mario Henrique Furlanetto Miranda ${ }^{3}$ Giulia Luiza Cecconello ${ }^{3}$ \\ ${ }^{1}$ Neurosurgeon, Hospital Regional do Oeste, Chapecó, SC, Brasil \\ 2 Sitting Member of the Brazilian Society of Neurosurgery - SBN, \\ São Paulo, SP, Brasil \\ Address for correspondence Marcelo Lemos Vieira da Cunha, MSc, \\ Hospital Regional do Oeste, Rua Lauro Muller, 224e, apto 501, Centro, CEP \\ 89801-600, Chapecó, SC, Brasil (e-mail: marcelolvc@yahoo.com.br).
}

${ }^{3}$ Medical Student, Universidade Comunitária da Região de Chapecó,

Chapecó, SC, Brasil

Arq Bras Neurocir 2017;36:178-184.

\begin{abstract}
Keywords

- neurilemmoma

- trochlear nerve

- diplopia

\section{Resumo}

\section{Palavras-chave \\ - neurilemoma \\ - nervo troclear \\ - diplopia}

Schwannomas arise from the Schwann cells of the peripheral and cranial nerves. They represent $8 \%$ of the primary cerebral neoplasms. Although schwannomas usually develop in sensory nerves, most often on the vestibular and trigeminal nerves, in very rare cases they can develop in motor nerves. We reported an unusual case of a 29-year-old woman with headache, nausea, vomiting, and blurred vision ongoing for 3 years. Magnetic resonance of the brain showed a solid-cystic expanded injury, heterogeneous, with limits partially defined and epicenter on the pineal gland. The lesion presented hyposignal in T1 and isosignal in T2. An intense enhancement of the solid part was observed after contrast injection. Foci of calcification and absence of diffusion restriction were also observed. The patient underwent microneurosurgery with supracerebellar infratentorial approach in a seated position. Subtotal resection was performed with maintenance of calcified tumor tissue adhered to the right Rosenthal basal vein. In the postoperative phase, the patient remained with diplopia when looking down; however, she reported improvement of headache and nausea.

Os schwannomas surgem das células de Schwann, dos nervos periféricos e cranianos e representam $8 \%$ das neoplasias cerebrais primárias. Apesar de os schwannomas se desenvolverem comumente em nervos sensoriais, mais frequentemente no nervo vestibular e trigêmeo, em casos muito raros ele pode se desenvolver em nervos motores. Relatamos um caso raro, de uma paciente do sexo feminino, 29 anos, com quadro de cefaleia, náuseas, vômitos e turvação visual há três anos. Ressonância magnética de encéfalo demonstrou lesão expansiva sólido-cística, heterogênea, com limites parcialmente definidos e epicentro na glândula pineal. Apresentou hipossinal em T1 e isossinal em T2. Houve intenso realce da parte sólida após injeção de contraste. Foram observados focos de calcificação e ausência de restrição à difusão. A paciente foi submetida a tratamento microneurocirúrgico com acesso infratentorial supracerebelar em posição sentada. Houve ressecção subtotal com manutenção de tecido tumoral calcificado aderido a veia basal de Rosenthal direita. No pós-operatório, a paciente permaneceu com diplopia na mirada ocular para baixo, entretanto apresentou melhora de cefaleia e náuseas.
\end{abstract}

received

March 5, 2017

accepted

May 2, 2017

published online

June 16, 2017
DOI https://doi.org/

10.1055/s-0037-1603919.

ISSN 0103-5355.
Copyright $\odot 2017$ by Thieme Revinter

Publicações Ltda, Rio de Janeiro, Brazil

License terms

ㄷ) (i) $\ominus$ (\$) 


\section{Introduction}

Schwannomas arise from the Schwann cells and represent 8\% of the primary cerebral neoplasms. ${ }^{1}$ They usually develop in sensory nerves, most often in vestibular nerve. ${ }^{2,3}$ In very rare cases schwannomas can develop in motor nerves, including the trochlear nerve. ${ }^{2}$ Trochlear nerve schwannomas are extremely rare and, according to our review, there are 37 surgical cases related in the literature, including ours.

\section{Case Report}

The case described is that of a female patient, 29 years-old, with headache associated with nausea, vomiting and blurred vision for 3 years. Imaging studies performed in another service showed an expansive lesion, causing mass effect, resulting in hydrocephalus. The patient was submitted to ventriculoperitoneal shunt, with improvement of the clinical condition. After the surgery, clinical follow-up was realized.

The patient was admitted in our service 2 years after the ventriculoperitoneal shunt surgery. She had diplopia when looking down, headache and nausea refractory to the clinical treatment. Magnetic resonance imaging (MRI) of the brain showed a solid-cystic expanded injury, heterogeneous, with limits partially defined and epicenter on the pineal gland. The lesion presented hyposignal in T1 and isosignal in T2. An intense enhancement of the solid part was observed after contrast injection (-Figs. 1, 2, 3). Foci of calcification and absence of diffusion restriction were observed as well.

Cerebrospinal fluid examination did not show tumor cells and the search for $\alpha$-fetoprotein and human chorionic gonadotropin were negative. The patient underwent microneurosurgery with supracerebellar infratentorial approach in a seated position. The surgery was performed with ultrasonic aspiration and central enucleation of the tumor, allowing a better mobility of the tumor capsule and dissection of deep veins and mesencephalic roof. Subtotal resection was performed, with maintenance of calcified tumor tissue adhered to the right Rosenthal basal vein due to profuse

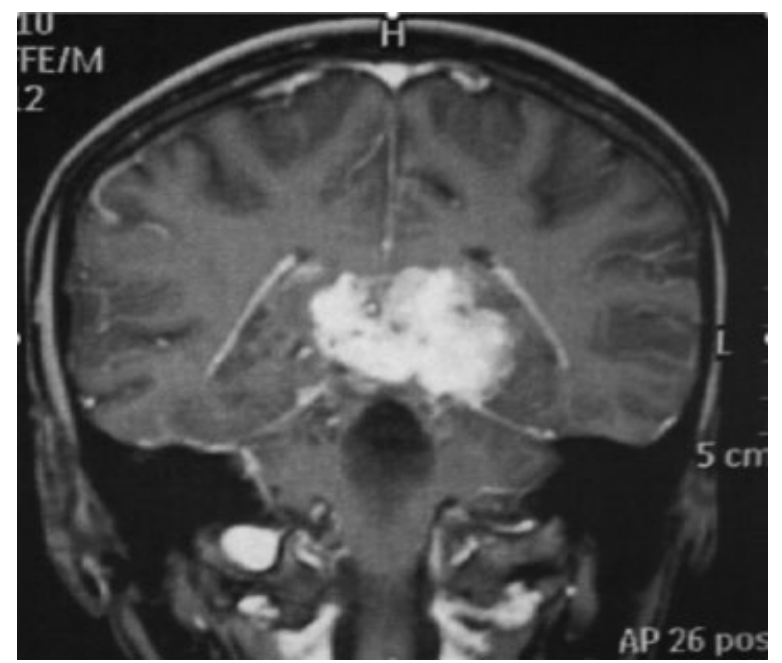

Fig. 1 Coronal cut in $\mathrm{T} 1$ after contrast injection with expansive lesion in the topography of the pineal gland.

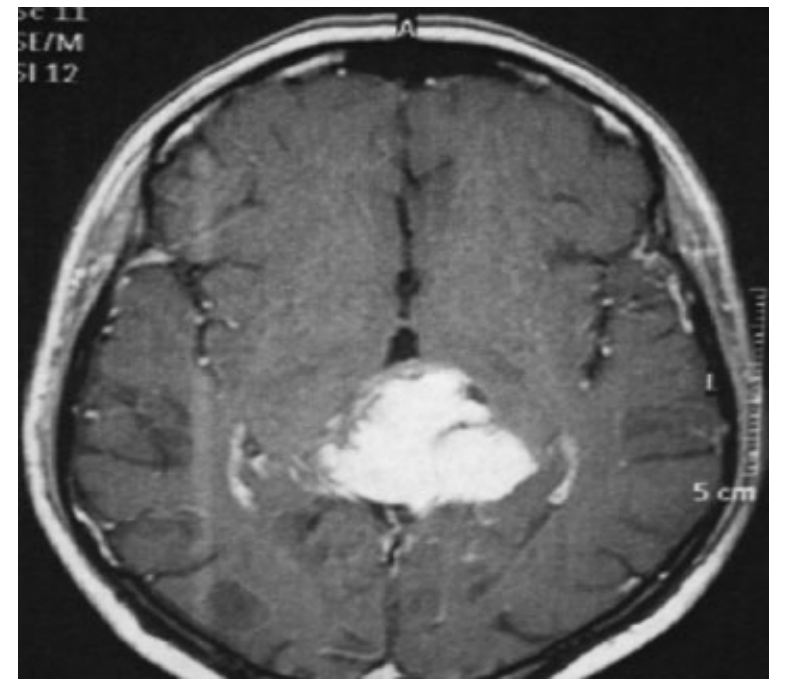

Fig. 2 Axial cut in $\mathrm{T} 1$ after injection of contrast with expansive lesion in the topography of the pineal gland.

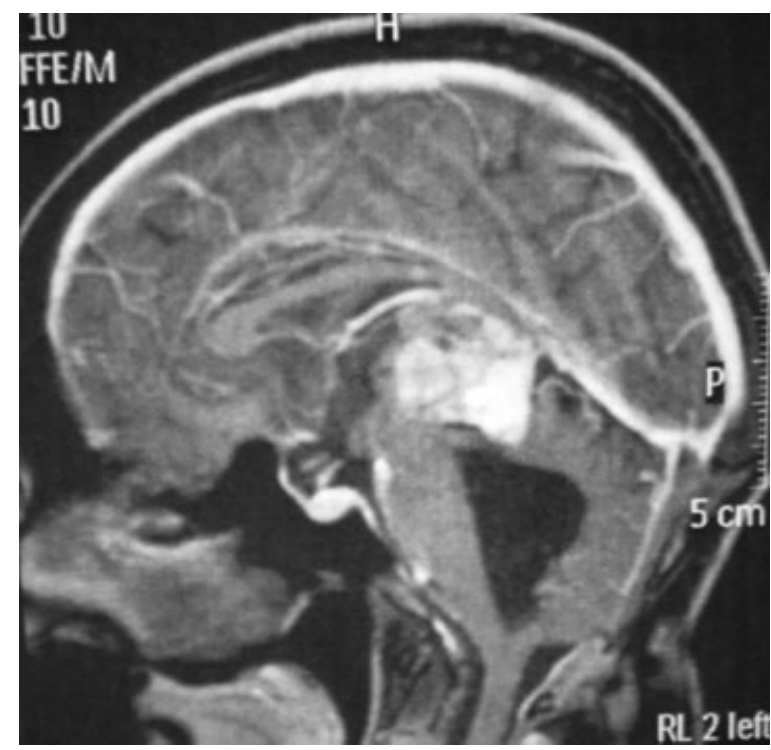

Fig. 3 Sagittal cut in $\mathrm{T} 1$ after injection of contrast with expansive lesion in the topography of the pineal gland.

bleeding when attempting resection of the tumor portion in this topography ( - Fig. 4).

The fourth nerve schwannomas typically show predominance of Antoni B tissue, have frequent clusters of xanthomatous macrophages and are poor in Verocay bodies (these are often more common in spinal tumors). Reticulin is abundant and distinctly pericellular in schwannomas.

Schwannomas are strongly and uniformly reactive for S-100 protein; they may be positive for epithelial membrane antigen (EMA), but not frquently, and do not exhibit the membrane-characteristic pattern of meningioma. Microscopic patterns and immunohistochemical findings in our case (-Fig. 5). In the postoperative phase, the patient remained with diplopia when looking down, reporting improvement of headache and nausea. 


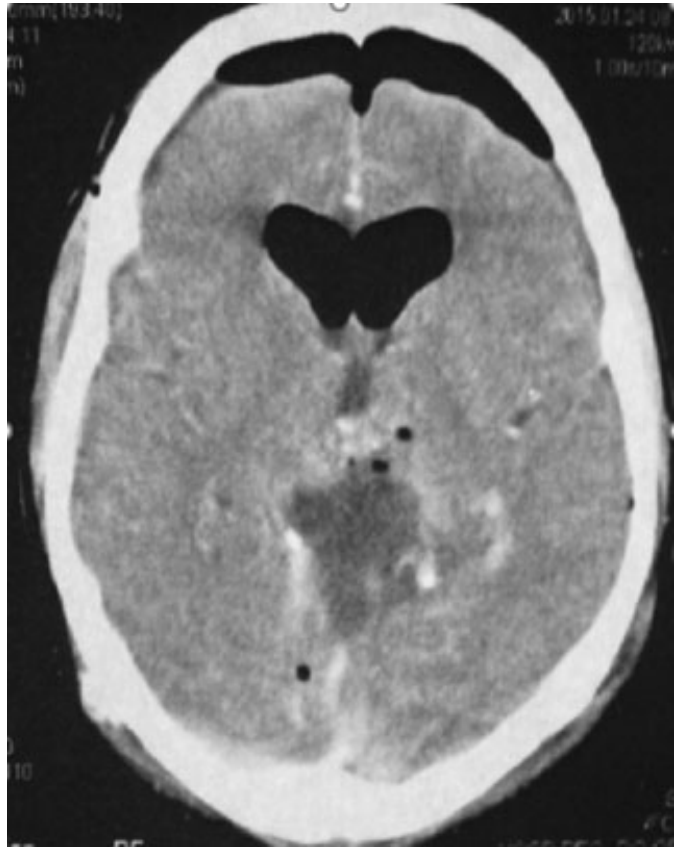

Fig. 4 Axial cut of computed tomography with contrast confirming postoperative status.

\section{Discussion}

We did a literary review using the PubMed and COCHRANE platforms searching for the following terms: trochlear nerve schwannoma and schwannoma surgery.

We found the occurrence of 37 surgical cases of trochlear schwannoma. The average age at diagnosis was 44.8 years, with a standard deviation of 15.1 . The female gender was predominant, with 21 cases (58.3\%) (-Table $\mathbf{1}$ ).

Trochlear nerve schwannoma presents as initial symptoms, in most cases, diplopia (60\%), hemiplegia (43\%), headache $(40 \%)$ and cerebellar symptoms (37\%), such as ataxia, dysmetria and nystagmus. ${ }^{29,33}$ In the present study, trochlear nerve palsy was evidenced in 17 (50\%) of 34 cases

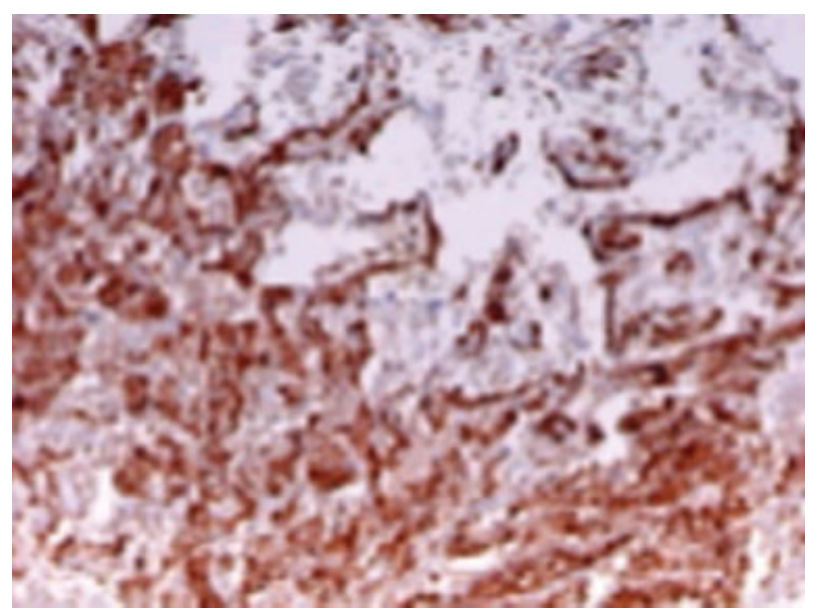

Fig. 5 Schwannoma strongly and uniformly reactive for S-100 protein by immunohistochemistry.
(-Table 1). In three case reports, intratumoral hemorrhage occurred as a complication. 7,26,33

Macroscopically, schwannomas are encapsulated, welldelimited, lobulated, generally grayish masses, and may have areas of cystic alterations and xanthomatosis. ${ }^{36,37}$

Magnetic resonance imaging showed to be the most used imaging exam in the evaluation of schwannoma. ${ }^{24,29,31,32,38}$ Computed tomography (CT) may also be used for the same purpose. ${ }^{33}$ At CT, it tends to present as a single, well-circumscribed and solid-appearing mass, located mainly in the course of the IV cranial nerve. ${ }^{32,38}$ At MRI, schwannomas present with hyposignal in T1, hypersignal in $\mathrm{T} 2$ and show an intense enhancement after intravenous administration of gadolinium contrast. ${ }^{38}$

Schwannomas can be diagnosed with conventional techniques when they are small and located in the cerebellumpontine cistern. However, when they reach very large sizes and the intracanalicular part is not obvious, it may be difficult to discriminate them from meningiomas and metastases. $^{39}$ So, the use of advanced imaging techniques may be necessary. Studies with vestibular schwannomas by the cerebellar-angle have shown that, through diffusion-weighted imaging (DWI), it is possible to observe that the schwannomas solid component, usually isointense to brain parenchyma, presents an apparent diffusion coefficient (ADC) that ranges from 1.1 to $1.7 \times 10-3 \mathrm{~mm}^{2} / \mathrm{S}$, which is considered elevated when compared with the normal brain parenchyma $\left(1.4 \times 10-3 \mathrm{~mm}^{2} / \mathrm{S}\right) .^{40,41}$ This increased coefficient may reflect the lower cell density of the Antoni B cells of schwannomas. However, the ADC alone cannot differentiate schwannoma from meningiomas. Even though schwannomas present a significantly higher average ADC value, there is considerable overlap among the values, which makes it difficult to differentiate meningiomas from schwannomas. ${ }^{41}$

Otherwise, it is possible to differentiate schwannomas from meningiomas through magnetic resonance (MR) perfusion. The schwannomas cerebral blood volume (CBV) is significantly smaller when compared with meningiomas. ${ }^{42}$ Even if there is an overlap in the reason between the CBV of both entities, a threshold of 4.4 is the highest found in the schwannomas, while the meningiomas CBV average ranges from 6 to 9. ${ }^{42-44}$

Another way to differentiate meningiomas from schwannomas is through MR proton spectroscopy, where the schwannomas show a myo-inositol peak of $3.55 \mathrm{pm}$ and absence of alanine. ${ }^{45}$ Meningiomas are characterized by a high choline signal with very low signs of creatine and $\mathrm{N}$-acetylaspartate, and alanine presence. ${ }^{46}$

The trochlear nerve schwannomas are classified in three subtypes: cisternal, cisternocavernous and cavernous. ${ }^{13}$ In our review, 34 of the 37 cases evaluated were cystenal (91.8\%), two cases (5.4\%) were cisternocavernous and one case $(2.7 \%)$ was cavernous.

Contrary to what can be found in the literature regarding trochlear schwannomas centered in the cisternal portion, the present case reports that the trochlear schwannoma was found with epicenter in the pineal gland. In our review, this only occurred in one other occasion, generating a second reported case with this approach for the referred pathology. The 


\begin{tabular}{|c|c|c|c|c|c|c|c|c|c|c|c|c|c|c|c|c|c|c|c|}
\hline 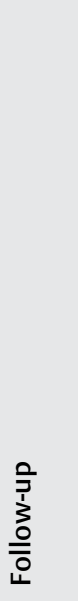 & 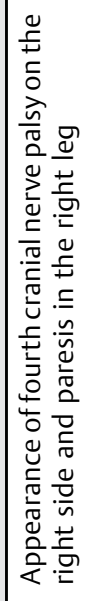 & 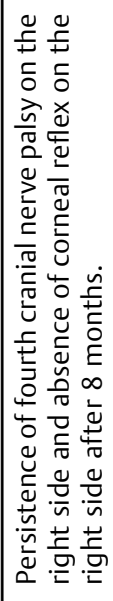 & 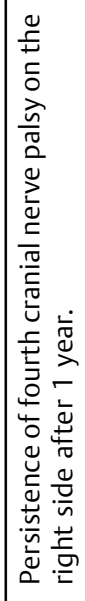 & 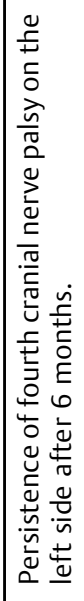 & 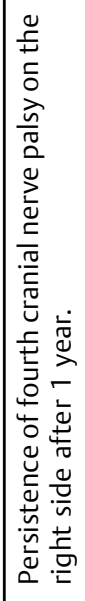 & 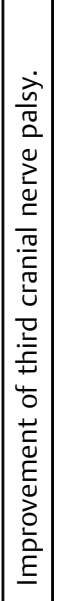 & 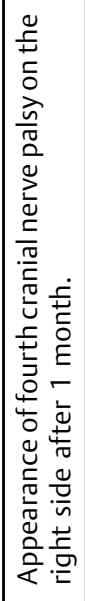 & 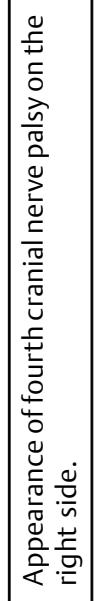 & 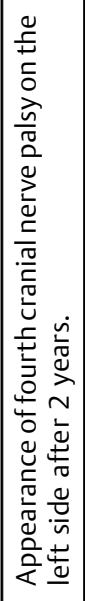 & 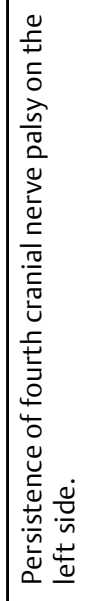 & 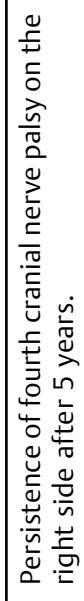 & $\bar{z}$ & 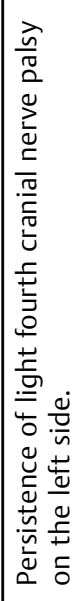 & 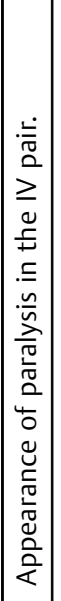 & 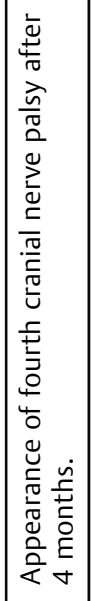 & 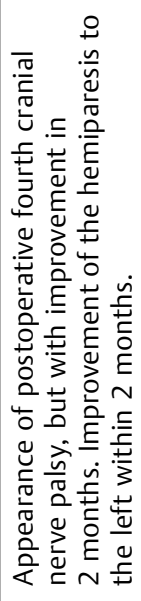 & 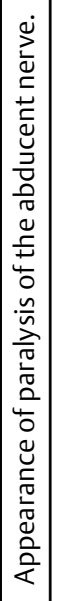 & 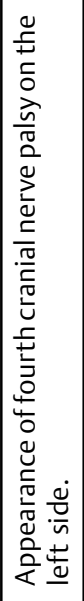 & 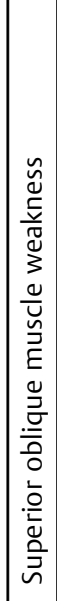 \\
\hline 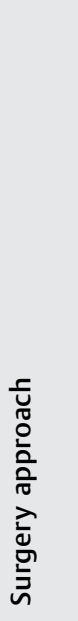 & 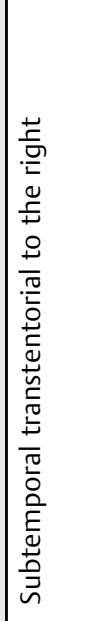 & 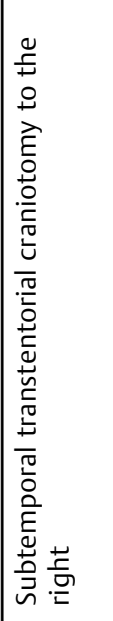 & 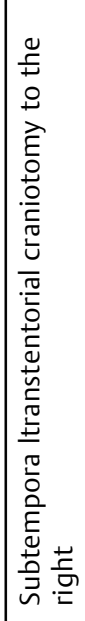 & 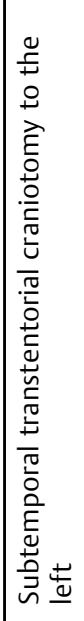 & 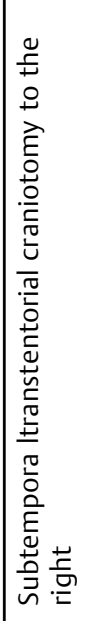 & 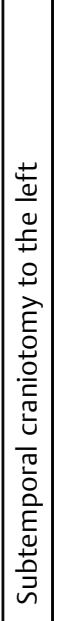 & 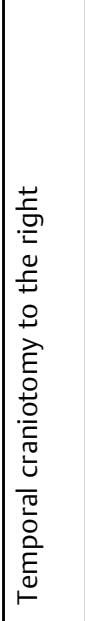 & 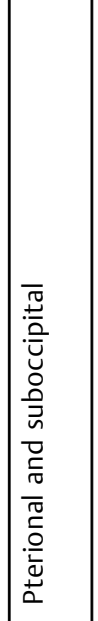 & 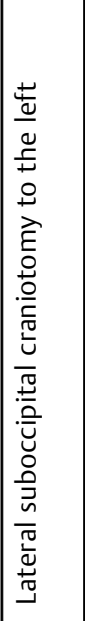 & 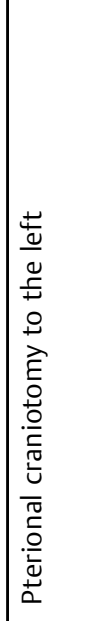 & 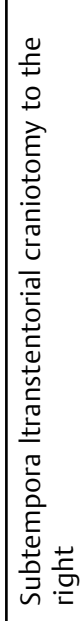 & $\bar{z}$ & 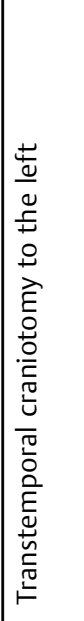 & 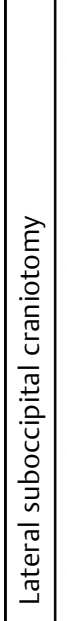 & 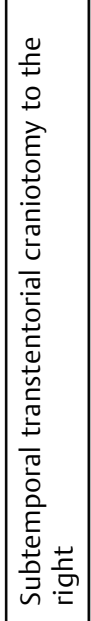 & 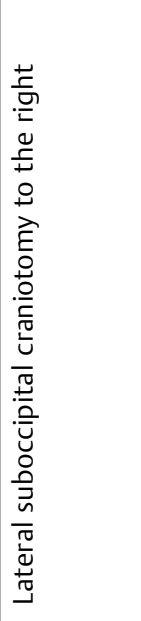 & 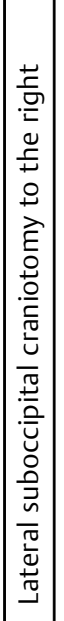 & 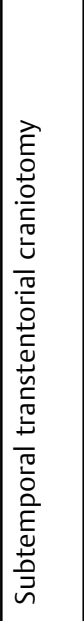 & 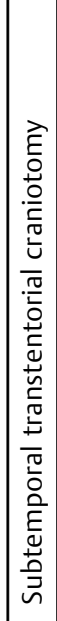 \\
\hline 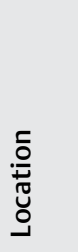 & 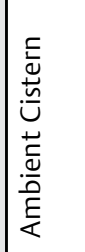 & 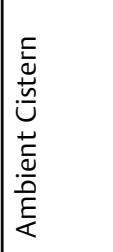 & 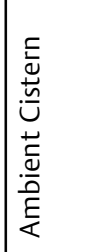 & 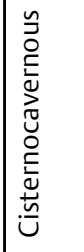 & 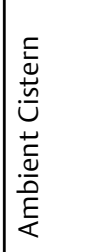 & 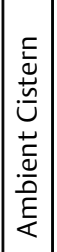 & 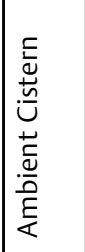 & 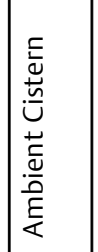 & 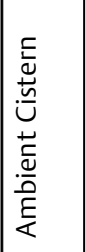 & 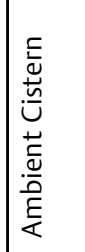 & 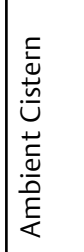 & 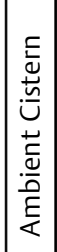 & 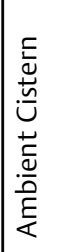 & 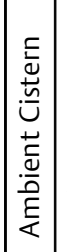 & 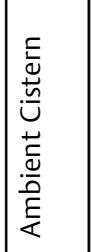 & 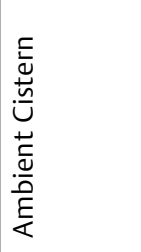 & 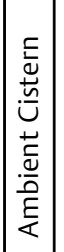 & 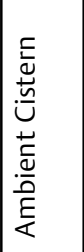 & 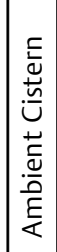 \\
\hline 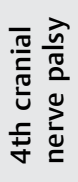 & 울 & $\stackrel{\check{\nu}}{\rightleftharpoons}$ & $\stackrel{\Xi}{\rightleftharpoons}$ & $\stackrel{\check{\nu}}{\rightleftharpoons}$ & $\stackrel{\check{\nu}}{\check{\nu}}$ & $\frac{0}{2}$ & 2 & 2 & 2 & $\stackrel{\check{y}}{\succ}$ & $\stackrel{\breve{u}}{\succ}$ & $\bar{z}$ & $\stackrel{\tilde{\nu}}{\succ}$ & in & $\frac{0}{2}$ & z & in & 2 & 2 \\
\hline 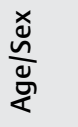 & $\frac{\omega}{\omega}$ & $\frac{\omega}{\sim}$ & 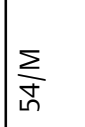 & $\frac{\omega}{6}$ & $\underset{m}{\stackrel{u}{r}}$ & $\frac{\omega}{\infty}$ & 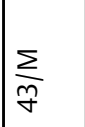 & $\frac{u}{\stackrel{N}{N}}$ & 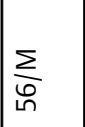 & $\frac{u}{m}$ & $\mid \sum_{i n}$ & $\bar{z}$ & $\frac{\omega}{0}$ & $\mid \begin{array}{l}2 \\
8 \\
0\end{array}$ & 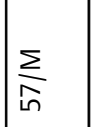 & $\sum_{\substack{\infty \\
0}}$ & $\sum_{0}^{0}$ & $\frac{\vec{u}}{\stackrel{\omega}{m}}$ & $\underset{\substack{\alpha \\
\infty}}{\stackrel{\omega}{\infty}}$ \\
\hline 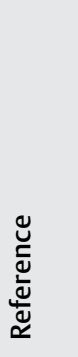 & $\mid \begin{array}{l}+ \\
\stackrel{\Xi}{\underline{\underline{I}}}\end{array}$ & 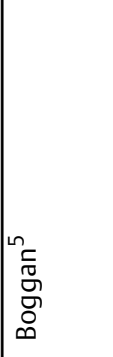 & 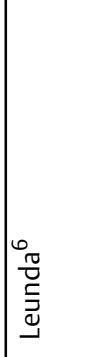 & 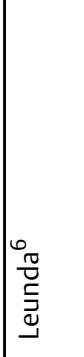 & 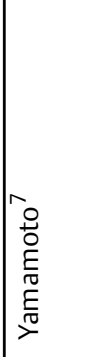 & 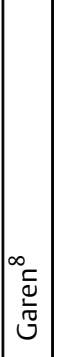 & 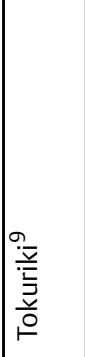 & 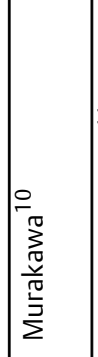 & 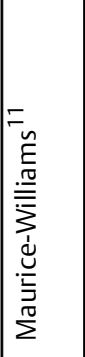 & 訔 & $\overline{\overline{\bar{u}}}$ & 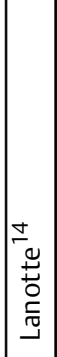 & 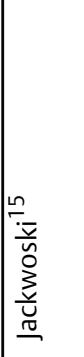 & {$\left[\begin{array}{l}0 \\
\dot{8} \\
\dot{\alpha}\end{array}\right]$} & 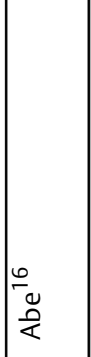 & 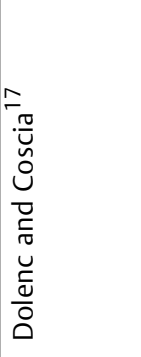 & $\mid \begin{array}{l}\infty \\
-\bar{\partial} \\
\overline{\bar{a}} \\
\infty \\
\infty\end{array}$ & 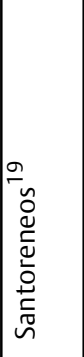 & 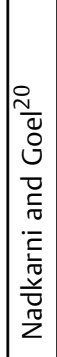 \\
\hline
\end{tabular}




\begin{tabular}{|c|c|c|c|c|c|c|c|c|c|c|c|c|c|c|c|c|c|c|}
\hline 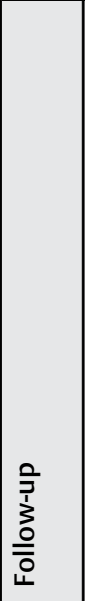 & 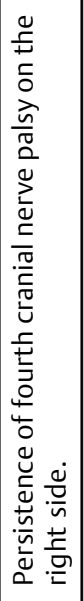 & 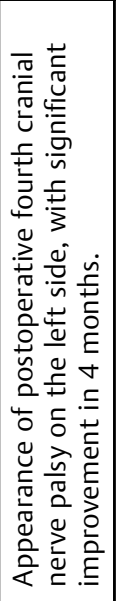 & 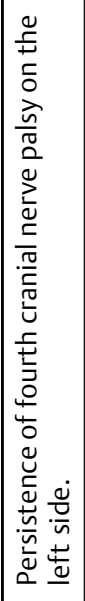 & 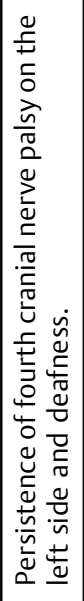 & 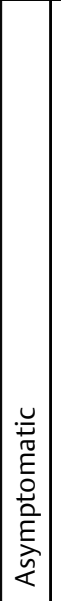 & 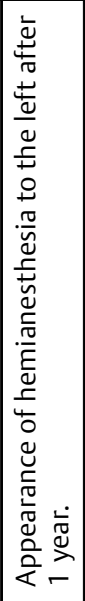 & 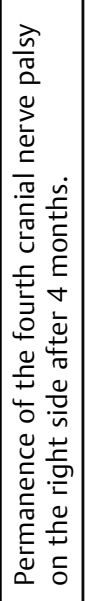 & 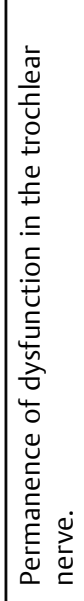 & \begin{tabular}{|l|}
$\mid$ \\
$\bar{z}$
\end{tabular} & $\bar{z}$ & 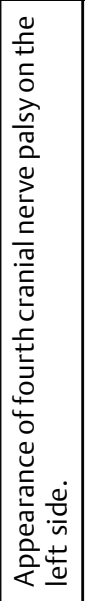 & 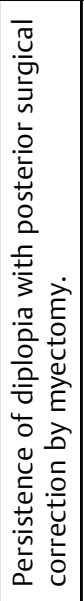 & 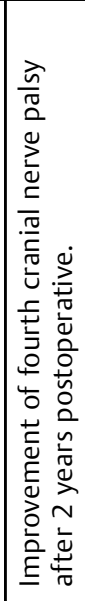 & 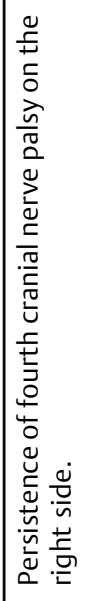 & 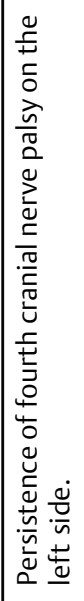 & 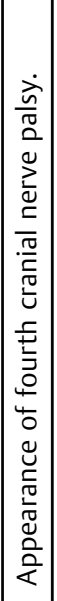 & 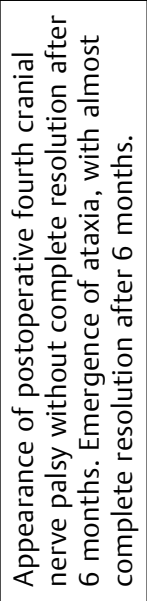 & 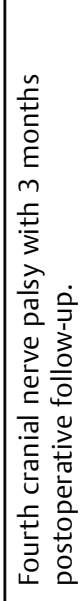 \\
\hline 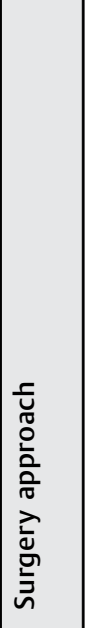 & 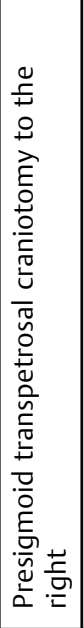 & 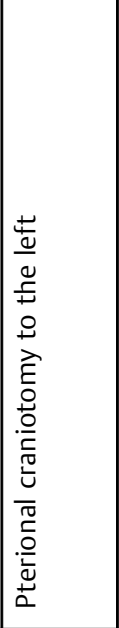 & 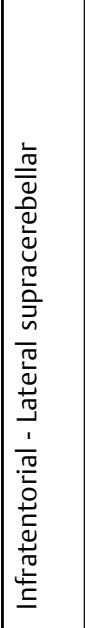 & 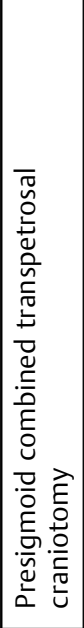 & 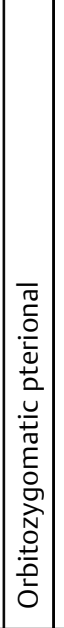 & 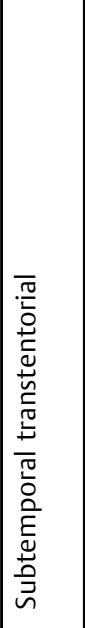 & 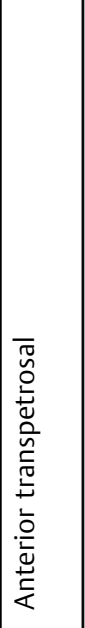 & 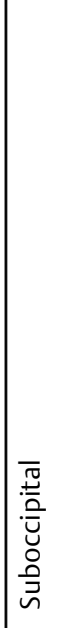 & 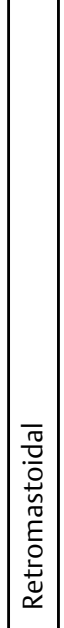 & 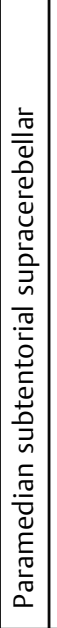 & 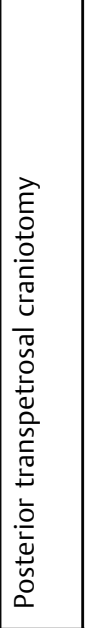 & 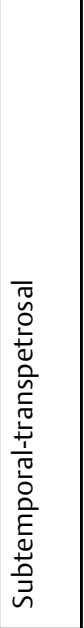 & 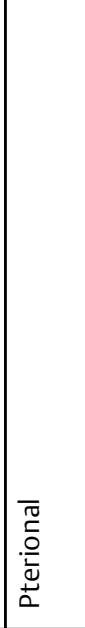 & 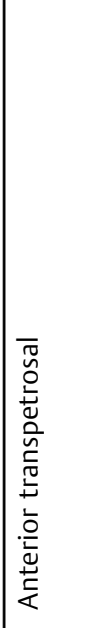 & 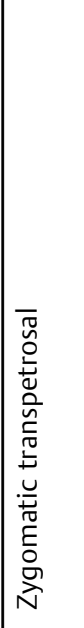 & 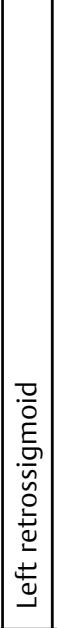 & 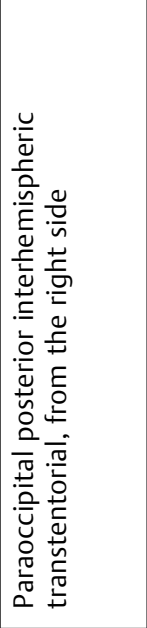 & 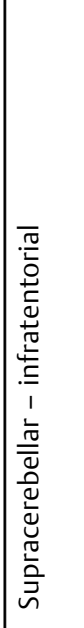 \\
\hline 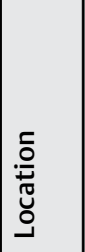 & 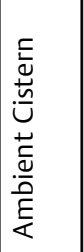 & 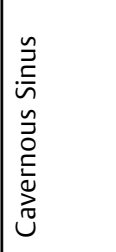 & 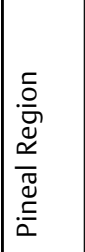 & 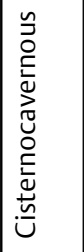 & 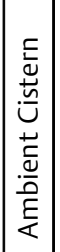 & 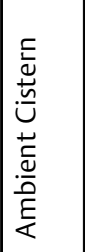 & 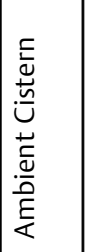 & 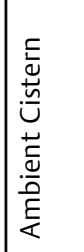 & 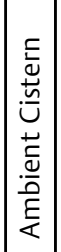 & 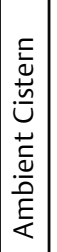 & 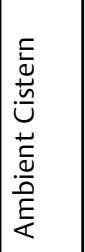 & 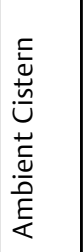 & 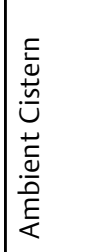 & 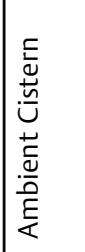 & 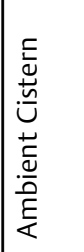 & 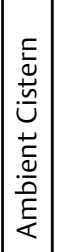 & 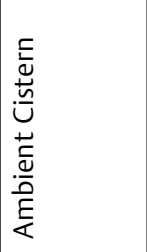 & 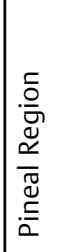 \\
\hline 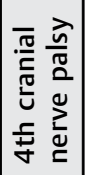 & $\stackrel{\check{\nu}}{\succ}$ & zo & $\stackrel{\check{\nu}}{\rightleftharpoons}$ & $\stackrel{\check{\nu}}{\nu}$ & 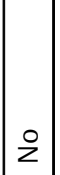 & z & $\stackrel{\check{\nu}}{\check{\nu}}$ & $\stackrel{\check{\nu}}{\rightleftharpoons}$ & $\bar{z}$ & $\bar{z}$ & zo & $\stackrel{\tilde{\nu}}{\searrow}$ & $\stackrel{\check{\nu}}{\rightleftharpoons}$ & $\stackrel{\tilde{\nu}}{\nu}$ & $\stackrel{y}{\nu}$ & i & i & $\stackrel{\check{\nu}}{\nu}$ \\
\hline 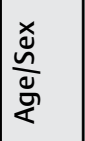 & $\frac{\Sigma}{\overline{6}}$ & 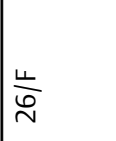 & $\frac{\sum}{m}$ & $\frac{\sum}{g}$ & $\frac{\stackrel{\Delta}{I}}{I}$ & $\frac{u}{\dot{y}}$ & $\sum_{\substack{\infty \\
\dot{j}}}$ & $\frac{\omega}{\sqrt{n}}$ & $\frac{\stackrel{\Delta}{\widehat{\gamma}}}{\vec{\gamma}}$ & $\frac{u}{\vec{y}}$ & $\frac{\stackrel{u}{\widehat{f}}}{\vec{f}}$ & $\begin{array}{l}\frac{\mathrm{u}}{\mathrm{O}} \\
\text { in }\end{array}$ & $\frac{\mathrm{L}}{\hat{0}}$ & $\sum_{f}$ & $\underset{\forall}{J}$ & $\frac{\Sigma}{\tilde{r}}$ & $\frac{\vec{U}}{\stackrel{\vec{t}}{\sim}}$ & $\frac{\frac{\omega}{2}}{N}$ \\
\hline 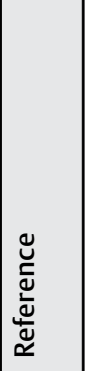 & 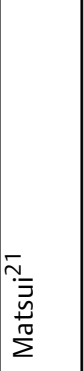 & 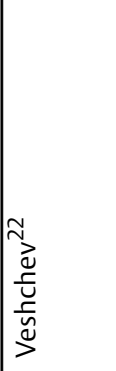 & 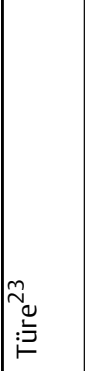 & 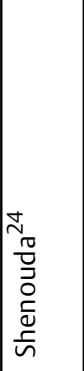 & $\stackrel{\sim}{z}$ & 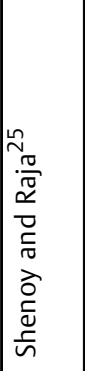 & 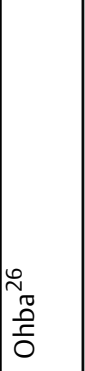 & 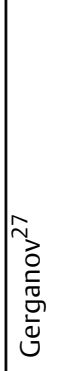 & 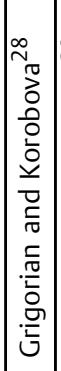 & 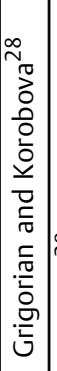 & 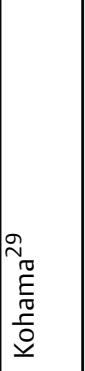 & 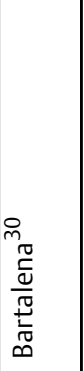 & $\begin{array}{l}\bar{m}_{\tilde{y}} \\
\stackrel{5}{0} \\
\partial\end{array}$ & 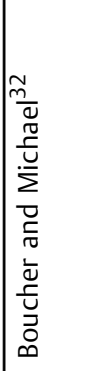 & 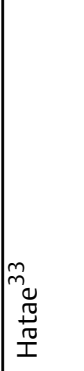 & 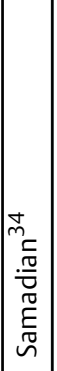 & 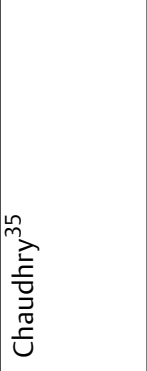 & 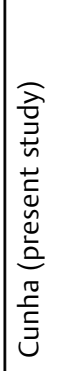 \\
\hline
\end{tabular}


incidence of tumors in the pineal region ranges from 0.4 to $1 \%$ of all primary brain tumors, with studies showing a higher percentage of tumors in this region in children and adolescents, with rates ranging from 3 to $8 \% .{ }^{47-51}$ As a differential diagnosis for the pineal region, we must consider the tumors divided in four categories: germ cell tumors, pineal parenchyma tumors, pineal gland support tissue tumors (for example, astrocytomas) and tumors originating from nearby structures (thalamic astrocytomas, tumors of Plexus choroid). ${ }^{52}$

The most common tumors of the pineal region are the germinative ones, responsible for 40 to $80 \%$ of the total number, with germinoma being the main representative. ${ }^{47,53}$ Tumors of the pineal parenchyma have a prevalence of 11 to $40 \%$ of pineal tumors, and can be divided into pineocytes, pineoblastomas, papillary tumors of the pineal region (PTPRs) and pineal parenchymal tumor of intermediate differentiation (PPTID). ${ }^{53,54}$

Tumors derived from supporting tissues or structures around the pineal gland include astrocytomas, ependymomas, papillomas and carcinomas of the choroid plexus, lymphomas, gangliogliomas, dermoid and epidermoid cysts, meningiomas and metastases. ${ }^{55}$ According to our literary review, our study is the second case of trochlear schwannoma that has its origin in the pineal region.

The therapeutic options for non-vestibular schwannomas include clinical observation through serial image analysis, microsurgery and stereotactic radiosurgery. ${ }^{56}$ Usually, when treating asymptomatic tumors, clinical observation may be a reasonable alternative, especially in elderly patients or those with other comorbidities, since these tumors usually have slow growth. ${ }^{56}$

According to our literature review, the most used surgical approach in the cases of trochlear schwannoma is the transtemperal subtemporal, performed in 13 of 36 cases, followed by transpetrosal (7 cases), lateral suboccipital ( 7 cases), pterional (5 cases), supracerebellar infratentorial ( 2 cases) supracerebellar subententorial (1 case), and paraoccipital trastentorial (1 case) (-Table 1). In one case, the surgical approach was not described. ${ }^{54}$ In our case, we used the intratentorial supracerebellar approach, the same one used in the Ture study. ${ }^{23}$ The surgical approach used in the previous case of trochlear schwannoma in the pineal gland was the posterior transtentorial interhemispheric paraoccipital. ${ }^{35}$

The postoperative of the previously reported cases demonstrated the difficulty and vulnerability in preserving the trochlear nerve during surgery (-Table 1). Among the 17 cases that did not present preoperative paralysis, 12 developed a new trochlear paralysis in the postoperative period, and in only 2 cases there were significant improvements in trochlear paralysis in the postoperative follow-up. From the 17 cases in which the patients had IV paresis, in only 1 trochlear function improvement happened, with diplopia improvement between 4 weeks and paralysis improvement after 2 years postoperatively. However, in this same case, the MRI 3 months after surgery showed a small remnant tumor. ${ }^{31}$

\section{Conclusion}

Trochlear nerve schwannomas are extremely rare; however, they generally have a good neurosurgical resectability. Our work presents an unusual trochlear nerve schwannoma, corresponding to the wide range of the possible pathological diagnoses when the region of the pineal gland is involved. In addition, our case also contrasts with the majority of the cases reported in the literature, which shows tumor epicenter furthest from nerve trajectory.

\section{References}

1 Russell DS, Rubinstein LJ. Pathology of tumors of the nervous system. 5th ed. Baltimore: Williams and Wilkins; 1989

2 Du R, Dhoot J, McDermott MW, Gupta N. Cystic schwannoma of the anterior tentorial hiatus. Case report and review of the literature. PediatrNeurosurg 2003;38(04):167-173

3 Ho KL. Schwannoma of the trochlear nerve. Case report. J Neurosurg 1981;55(01):132-135

4 King JS. Trochlear nerve sheath tumor; case report. J Neurosurg 1976;44(02):245-247

5 Boggan JE, Rosenblum ML, Wilson CB. Neurilemmoma of the fourth cranial nerve. Case report. J Neurosurg 1979;50(04):519-521

6 Leunda G, Vaquero J, Cabezudo J, Garcia-Uria J, Bravo G. Schwannoma of the oculomotor nerves. Report of four cases. J Neurosurg 1982;57(04):563-565

7 Yamamoto M, Jimbo M, Ide M, Kubo O. Trochlear neurinoma. SurgNeurol 1987;28(04):287-290

8 Garen PD, Harper CG, Teo C, Johnston IH. Cystic schwannoma of the trochlear nerve mimicking a brain-stem tumor. Case report. J Neurosurg 1987;67(06):928-930

9 Tokuriki Y, Yamashita J, Kikuchi H, Asato R, Handa H. Trochlear nerve neurinoma-case report. Neurol Med Chir (Tokyo) 1988; 28(01):70-73 [Tokyo]

10 Murakawa T, Sakai H, Ueda T, Yokoyama K, Takada M, Yamada H. Trochlear neurinoma: case report. Takayama Red Cross HospKiyou 1988;12:163-167

11 Maurice-Williams RS. Isolated schwannoma of the fourth cranial nerve: case report. J NeurolNeurosurg Psychiatry 1989;52(12): 1442-1443

12 Samii M, Draf W, Lang J. Surgery of the Skull Base: An Interdisciplinary Approach. Berlin: Springer; 1989 pp. 335-7

13 Celli P, Ferrante L, Acqui M, Mastronardi L, Fortuna A, Palma L. Neurinoma of the third, fourth, and sixth cranial nerves: a survey and report of a new fourth nerve case. SurgNeurol 1992;38(03): 216-224

14 Lanotte M, Giordana MT, Forni C, Pagni CA. Schwannoma of the cavernous sinus. Case report and review of the literature. J NeurosurgSci 1992;36(04):233-238

15 Jackowski A, Weiner G, O'Reilly G. Trochlear nerve schwannomas: a case report and literature review. Br J Neurosurg 1994;8(02): 219-223

16 Abe T, Iwata T, Shimazu M, et al. [Two cases of trochlear nerve neurinoma]. No ShinkeiGeka 1994;22(04):371-375

17 Dolenc VV, Coscia S. Cystic trochlear nerve neurinoma. $\mathrm{Br} \mathrm{J}$ Neurosurg 1996;10(06):593-597

18 Beppu T, Yoshida Y, Wada T, et al. Trochlear and abducens nerve neurinomas accompanied by a cerebellopontine angle meningioma-case report. Neurol Med Chir (Tokyo) 1997;37(05):416-421

19 Santoreneos S, Hanieh A, Jorgensen RE. Trochlear nerve schwannomas occurring in patients without neurofibromatosis: case report and review of the literature. Neurosurgery 1997;41(01): 282-287 
20 Nadkarni TD, Goel A. Trochlear nerve neurinoma presenting as pathological laughter. Br J Neurosurg 1999;13(02):212-213

21 Matsui T, Morikawa E, Morimoto T, Asano T. Presigmoidtranspetrosal approach for the treatment of a large trochlear nerve schwannoma-case report. Neurol Med Chir (Tokyo) 2002; 42(01):31-35

22 Veshchev I, Spektor S. Trochlear nerve neuroma manifested with intractable atypical facial pain: case report. Neurosurgery 2002; 50(04):889-891, discussion 891-892

23 Türe U, Ozduman K, Elmaci I, Pamir MN. Infratentorial lateral supracerebellar approach for trochlear nerve schwannoma. J ClinNeurosci 2002;9(05):595-598

24 Shenouda EF, Ghosh A, Coakham HB. Trochlear nerve schwannoma removed by combined petrosal approach. Br J Neurosurg 2002;16(06):600-604

25 Shenoy SN, Raja A. Cystic trochlear nerve neurinoma mimicking intrinsic brainstem tumour. Br J Neurosurg 2004;18(02):183-186

26 Ohba S, Miwa T, Kawase T. Trochlear nerve schwannoma with intratumoral hemorrhage: case report. Neurosurgery 2006;58 (04):E791, discussion E791

27 Gerganov V, Amir S, Koerbel A, Brandes A, Stan A, Madjid S. Cystic trochlear nerve schwannoma. Case report. SurgNeurol 2007;68 (02):221-225

28 Grigorian IuA, Korobova AN. [Neurinomas of the trochlear nerve]. Zh Vopr Neirokhir Im N N Burdenko 2008;(01):50-52

29 Kohama M, Murakami K, Endo T, Watanabe M, Tominaga T. Surgical and histological observations of trochlear neurinoma: case report. Neurol Med Chir (Tokyo) 2009;49(05):217-220

30 Bartalena T, Leoni C, Trossello MP, et al. Hourglass cystic schwannoma of the trochlear nerve. Acta Biomed 2010;81(02):147-150

31 Younes WM, Hermann EJ, Krauss JK. Cisternal trochlear nerve schwannoma: improvement of diplopia after subtotal tumour excision. Br J Neurosurg 2012;26(01):107-109

32 Boucher AB, Michael LM II. The middle fossa approach for the removal of a trochlear schwannoma. Case Rep Neurol Med 2014; 2014(01):672314

33 Hatae R, Miyazono M, Kohri R, Maeda K, Naito S. Trochlear nerve schwannoma with intratumoral hemorrhage presenting with persistent hiccups: a case report. J NeurolSurg Rep 2014; 75(01):e183-e188

34 Samadian M, Farzin N, Bakhtevari MH, Hallajnejad M, Rezaei O. Isolated trochlear nerve schwannoma presenting with diplopia: A case report and literature review. Interdisciplinary Neurosurgery 2015;2(02):111-114

35 Chaudhry NS, Ahmad FU, Morcos JJ. Pineal region schwannoma arising from the trochlear nerve. J ClinNeurosci 2016;32:159-161

36 Stull MA, Moser RP Jr, Kransdorf MJ, Bogumill GP, Nelson MC. Magnetic resonance appearance of peripheral nerve sheath tumors. Skeletal Radiol 1991;20(01):9-14

37 al-Ghamdi S, Black MJ, Lafond G. Extracranial head and neck schwannomas. J Otolaryngol 1992;21(03):186-188

38 Feinberg AS, Newman NJ. Schwannoma in patients with isolated unilateral trochlear nerve palsy. Am J Ophthalmol 1999;127(02): 183-188

39 Press GA, Hesselink JR. MR imaging of cerebellopontine angle and internal auditory canal lesions at 1.5 T. AJR Am J Roentgenol 1988; 150(06):1371-1381
40 Sener RN. Diffusion magnetic resonance imaging of solid vestibular schwannomas. J Comput Assist Tomogr 2003;27(02): 249-252

41 Yamasaki F, Kurisu K, Satoh K, et al. Apparent diffusion coefficient of human brain tumors at MR imaging. Radiology 2005;235(03): 985-991

42 Hakyemez B, Erdogan C, Bolca N, Yildirim N, Gokalp G, Parlak M. Evaluation of different cerebral mass lesions by perfusionweighted MR imaging. J MagnReson Imaging 2006;24(04): 817-824

43 Kremer S, Grand S, Rémy C, et al. Contribution of dynamic contrast MR imaging to the differentiation between dural metastasis and meningioma. Neuroradiology 2004;46(08):642-648

44 Yang S, Law M, Zagzag D, et al. Dynamic contrast-enhanced perfusion MR imaging measurements of endothelial permeability: differentiation between atypical and typical meningiomas. AJNR Am J Neuroradiol 2003;24(08):1554-1559

45 Cho YD, Choi GH, Lee SP, Kim JK. (1)H-MRS metabolic patterns for distinguishing between meningiomas and other brain tumors. MagnReson Imaging 2003;21(06):663-672

46 Majós C, Julià-Sapé M, Alonso J, et al. Brain tumor classification by proton MR spectroscopy: comparison of diagnostic accuracy at short and long TE. AJNR Am J Neuroradiol 2004;25(10): 1696-1704

47 Hoffman HJ, Yoshida M, Becker LE, Hendrick EB, Humphreys RP; Experience at the Hospital for Sick Children. Pineal region tumors in childhood. Experience at the Hospital for Sick Children. 1983. PediatrNeurosurg 1994;21(01):91-103, discussion 104

48 Russell DS, Rubinstein LJ. Pathology of Tumors of the Nervous System. 4th ed. Baltimore: Wiliams\& Wilkins; 1977

49 Surawicz TS, McCarthy BJ, Kupelian V, Jukich PJ, Bruner JM, Davis FG. Descriptive epidemiology of primary brain and CNS tumors: results from the Central Brain Tumor Registry of the United States, 1990-1994. Neuro-oncol 1999;1(01):14-25

50 Kaatsch P, Rickert CH, Kühl J, Schüz J, Michaelis J. Populationbased epidemiologic data on brain tumors in German children. Cancer 2001;92(12):3155-3164

51 Dolecek TA, Propp JM, Stroup NE, Kruchko C. CBTRUS statistical report: primary brain and central nervous system tumors diagnosed in the United States in 2005-2009. Neuro-oncol 2012; 14(5, Supp 15):v1-v49

52 Smith AB, Rushing EJ, Smirniotopoulos JG. From the archives of the AFIP: lesions of the pineal region: radiologic-pathologic correlation. Radiographics 2010;30(07):2001-2020

53 Hirato J, Nakazato Y. Pathology of pineal region tumors. J Neurooncol 2001;54(03):239-249

54 Louis DN, Ohgaki H, Wiestler OD, et al. The 2007 WHO classification of tumours of the central nervous system. ActaNeuropathol 2007;114(02):97-109

55 Regis J, Bouillot P, Rouby-Volot F, Figarella-Branger D, Dufour H, Peragut JC. Pineal region tumors and the role of stereotactic biopsy: review of the mortality, morbidity, and diagnostic rates in 370 cases. Neurosurgery 1996;39(05):907-912, discussion 912-914

56 Pollock BE, Foote RL, Stafford SL. Stereotactic radiosurgery: the preferred management for patients with nonvestibular schwannomas? Int J RadiatOncolBiol Phys 2002;52(04):1002-1007 